29- Rektumkarzinom: Erweiterte Chirurgie

\begin{tabular}{ll|l} 
Prof. Dr. med. & $\begin{array}{l}\text { Prof. Dr. med. } \\
\text { Dr. h.c. K.-W. Jauch } \\
\text { Direktor der Chirur- } \\
\text { gischen Klinik und } \\
\text { Poliklinik Großhadern } \\
\text { LMU München }\end{array}$ & $\begin{array}{l}\text { Martin E. Kreis } \\
\text { Leitender Oberarzt } \\
\text { Chirurgische Klinik und } \\
\text { Poliklinik Großhadern } \\
\text { LMU München }\end{array}$ \\
\hline
\end{tabular}

31- Rektumkarzinom: Neoadjuvante Radiochemotherapie

34- Kolonkarzinom: Adjuvante Chemotherapie und Nachsorge

\title{
Differenzierte Therapiekonzepte sind Standard
}

— Das kolorektale Karzinom ist mit 70000 Neuerkrankungen der häufigste gastrointestinale Tumor in Deutschland und sowohl bei Männern als auch bei Frauen der zweithäufigste bösartige Tumor überhaupt. Die Behandlung war viele Jahrzehnte ausschließlich eine Domäne der Chirurgie, da bei einem Teil der Patienten nur durch die Operation in einem frühen Stadium ein Langzeitüberleben sichergestellt werden konnte. In den letzten 20 Jahren hat sich bei der Behandlung des fortgeschrittenen Rektumkarzinoms jedoch ein erheblicher Wandel vollzogen, ausgeprägter noch als beim Kolonkarzinom. Beim Rektumkarzinom kam es zu einer erheblichen Differenzierung der chirurgischen Therapie: lokale Exzision mittels transanaler endoskopischer Mikrochirurgie, Verbesserung der OP-Technik mit totaler mesorektaler Exzision, Sphinktererhalt in fast 90\% der Fälle, PouchRekonstruktionen sowie in letzter Zeit Verbesserung der Technik der abdomino-perinealen Rektumexstirpation als zylindrische Resektion. Mit der Strahlen-, Chemo- und „targeted“" Therapie verfügen wir über weitere sehr wirksame Modalitäten.

Die Entwicklung bei der neoadjuvanten Radiochemotherapie, die der Strahlentherapie zunehmende Bedeutung verschaffte, hat sicherlich zur Verbesserung der onkologischen Ergebnisse beigetragen. Dabei ist aber zu beachten, dass diese Behandlungsform ebenso wie die Chirurgie mit erheblichen Folgen für den Patienten einhergeht. Die liberale Praxis in Deutschland, die derzeit zur neoadjuvanten Radiochemotherapie bei $80 \%$ aller Rektumkarzinome führt, sollte deshalb stufenweise überprüft werden, wenngleich die grundsätzliche Bedeutung der Radiochemotherapie und der damit verbundene onkologische Benefit für den Patienten nicht infrage steht.

\section{Behandlung erfolgreicher, aber komplexer}

Die chirurgischen Möglichkeiten sind heute vielfältig, darüber hinaus bestehen multimodale Behandlungsoptionen. Mit moderner Bildgebung und entsprechender Expertise wird eine solide präoperative Kategorisierung des Tumorstadiums herbeigeführt. So hat sich die Behandlung des Rektumkarzinoms von einer ehemals

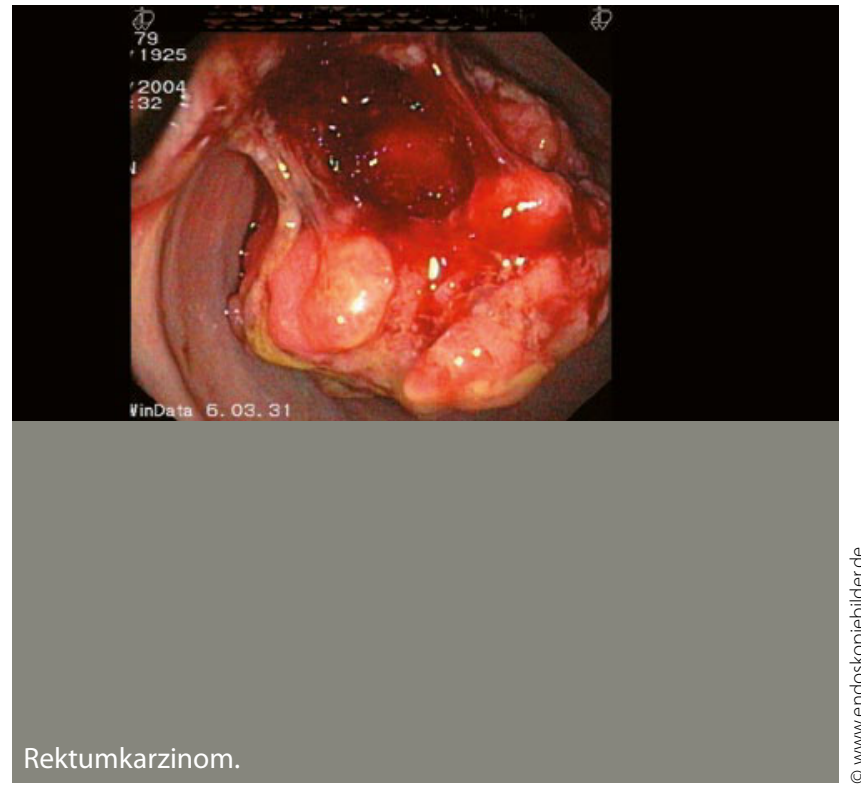

ausschließlich chirurgischen Therapie, die jeder Allgemeinchirurg im Repertoire hatte, zu einer Behandlung gewandelt, die eine erhebliche Spezialisierung fordert, um exzellente Ergebnisse zu erreichen. Dabei ist die Sicherstellung der multimodalen Therapie an Ressourcen gekoppelt, die heutzutage typischerweise in entsprechenden Darmkrebszentren vorgehalten werden. Diese Entwicklung ist nicht ohne Erfolg geblieben. Insbesondere im Raum München konnte durch das Tumorregister dokumentiert werden, dass sich die früher deutlich schlechtere Prognose des Rektumkarzinoms heute an die Prognose des Kolonkarzinoms angeglichen hat.

Dieser Fortbildungsschwerpunkt soll Ihnen für die Führung Ihrer Patienten, die an einem kolorektalen Karzinom erkrankt sind, fundierte Hindergrundinformationen liefern und eine Hilfestellung für deren Betreuung und Behandlung geben. 